Plant and Soil 137: 167-170, 1991.

(C) 1991 Kluwer Academic Publishers. Printed in the Netherlands.

PLSO NL26

\title{
The Anabaena-Azolla symbiosis: Diversity and relatedness of neotropical host taxa
}

\author{
W.J. ZIMMERMAN ${ }^{1}$, I. WATANABE ${ }^{2}$ and T.A. LUMPKIN ${ }^{3}$ \\ ${ }^{1}$ Department of Natural Sciences, University of Michigan-Dearborn, Dearborn, MI 48128, USA, ${ }^{2}$ Soil \\ Microbiology Department, International Rice Research Institute, P.O. Box 933, Manila, Philippines and \\ ${ }^{3}$ Department of Agronomy and Soils, Washington State University, Pullman, WA 99164, USA
}

Key words: azolla, DNA polymorphisms, isoenzymes, taxonomy

\begin{abstract}
The Anabaena-Azolla association has proved to be an effective biofertilizer in tropical regions of wetland rice production. Three neotropical host species, $A$. microphylla, $A$. caroliniana, and $A$. mexicana, are similar in vegetative morphology (growth habits, frond dimensions, trichome cell number) and ecophysiology (relative heat tolerance). They were observed during our investigation to also be genetically alike and distinct from other taxa.
\end{abstract}

\section{Introduction}

A major constraint to Anabaena-Azolla utilization as a rice biofertilizer is the inability to distinguish strains and even some species of the host. There are seven designated species of Azolla, an aquatic fern, divided by secondary reproductive features into two sections. Species traits in Section Azolla have been historically confined to anatomical characters and are of little value among neotropical taxa (three of five species). Varietal separation has never been achieved.

The morphological similarity of cultivated accessions has always been a major obstacle to the proper care of germplasm. Maintenance of live biomass is necessary since Azolla accessions rarely sporulate in culture collections. Fingerprinting assists the selection of isolates for field use, provides a means to monitor cross-contamination and somaclonal variation, and facilitates identification of some species. In this paper, we review our isoenzyme and DNA fingerprinting results thus far with azolla.
Species code

A. filiculoides FI A. pinnata $\mathrm{P}^{\prime \prime}$

A. rubra RU A. pinnata v. pinnata PP

A. caroliniana $\mathrm{CA}$ A. pinnata v. imbricata PI

A. microphylla $\mathrm{MI} A$. nilotica NI

A. mexicana $\mathrm{ME}$

\section{Materials and methods}

Isoenzyme comparisons by gel electrophoresis were first completed using known protocols (Zimmerman et al., 1989a,b). For DNA comparisons, genomic DNA digestions, fractionation, blotting, and Southern hybridizations were completed by standard techniques (Maniatis et al., 1983). Numerous gene probes were obtained for hybridizations. Thirty heterologous probes from maize, pea, lentil, squash, rice, soybean, Clarkia, barley, tomato, lettuce and the bacteriophage M13 were tested. A genomic DNA library (fragment sizes from $0.5-2 \mathrm{~kb}$ ) was also made from an ME strain and partially screened. Another homologous probe, a $2.3 \mathrm{~kb}$ Hind III 
insert in pBS, from MI 431 (FAAS) was donated courtesy of J. Plazinski (Plazinski et al., 1989).

\section{Results and discussion}

Isozymes

Two initial objectives were achieved (Van Cat et al., 1989; Zimmerman et al., 1989d): 1) zymograms of healthy vs stressed leaf tissue, leaf vs root tissue, and leaf tissue with or without the cyanobiont were compared for discrepancies, and 2) the potential of this methodology for hybrid identification in Azolla was tested. These objectives were followed by a mass screening of accessions. Banding patterns for New World accessions were scored. The fingerprinting results were then interpreted from a perspective of biochemical taxonomy within Section Azolla (Zimmerman et al., 1989b).

Healthy leaves yielded more extensive information in their zymograms than did stressed leaves. Phosphorus deficiency was chosen since it is a common field stress and the most important limiting nutrient for Azolla. A reduced staining intensity of enzymes or the lack of visualization of loci was often observed in the zymograms of P-deficient fronds. The presence of the cyanobiont in leaves did not influence banding patterns (comparing FI I Anabaena azollae). Root zymograms of PI were found to be unlike those of leaves for two of four tested enzymes. Additionally, the presence of phenolics and other root compounds interfered with the resolution of zymograms.

Hybrids of $\mathrm{FI} \times \mathrm{MI}$ were successfully confirmed by observing the patterns of certain multiloci enzymes such as PGM or SKDH. New hybrids (including those from crosses of $\mathrm{ME} \times \mathrm{MI}$ ) continue to be confirmed at IRRI by enzyme electrophoresis,

The subsequent mass screening of accessions within this section was combined with data garnered from trichome morphology prior to final species analyses. Twelve enzymes were stained. Nine contained 17 polymorphic loci and 126 allelomorphic characters; three (ALD, G6PDH, F1, 6DP) appeared to be monomorphic. At least two loci each were present in SKDK and 6PGD.
FI accessions were enzymatically distinctive (e.g., IDH) and also identified by prominent one-celled leaf trichomes. True RU accessions were found only in Australia and New Zealand, and possessed barely discernable trichomes. The Japanese RU was concluded to actually be FI. Probable duplicated entries (e.g., IRRI strains 2010/2022, 1005/1006/SWD, 1010/1016) and other enzymatically identical accessions (6007/ $6008,6003 / 6005 / 6006,2016 / 2018$ ) were reported. Unique strains were recorded, both those positively identified to species such as FI and RU and others of the CA-ME-MI cluster (e.g., 2001, 2009, 2021, UCD 60 (probably IRRI 2005), 3001, 3008, 3016, 4033).

MI (from Paraguay and the Galapagos Islands) were all unique by their faster PGM-2 locus. However, a principal component analysis showed no discernible phenetic distance separating MI from the other species of the CA-ME-MI cluster (Fig. 1). The strains of these species commonly have two-celled leaf trichomes.

Incorrect labelling was found among many isolates of the 1000 series (FI) in the IRRI collection. Colombian strains within the 10141027 series, among others, have now been relabelled as ME (2010-2022). An earlier series of growth experiments under high temperature had indicated that these strains were heat-tolerant

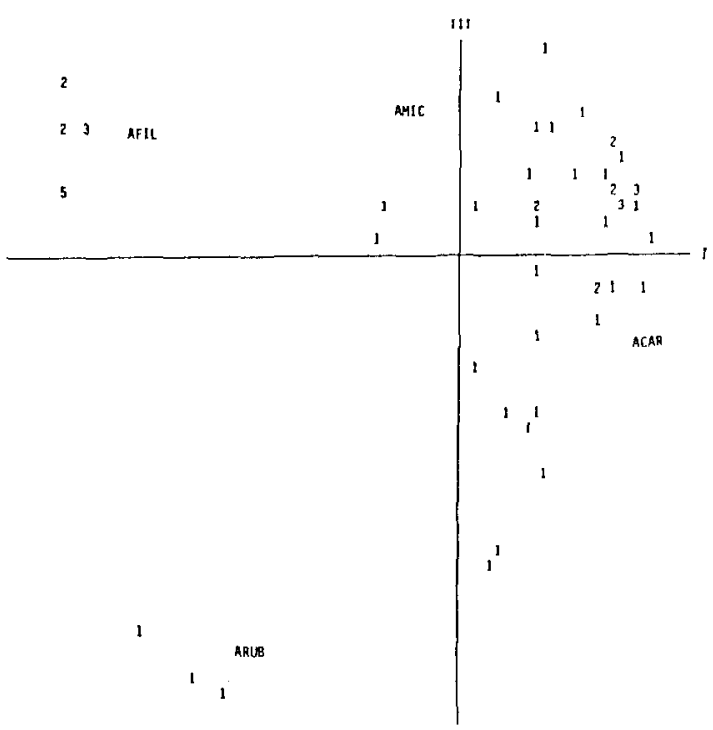

Fig. 1. Principal component analysis of 57 accessions of New World Azolla (Zimmerman et al., 1989b). AFIL $=\mathrm{FI}$, $\mathrm{ARUB}=\mathrm{RV}, \mathrm{AMIC}=\mathrm{MI}, \mathrm{ACAR}=\mathrm{CA}, \mathrm{ME}$. 
(IRRI, 1986). Biomass production was similar to MI 4018 and unlike heat-sensitive FI. Possible natural hybrids in this series were noted for further genetic analysis.

\section{DNA polymorphisms}

Results with DNA restriction fragment length polymorphisms (RFLPs) reinforced the conclusions derived from phenetic data and located additional polymorphisms among strains which were enzymatically related. Heterologous/ homologous gene probes and detailed hybridization results are described elsewhere (submitted manuscripts).

Hybridization patterns in several cases distinguished $\mathrm{P}^{\prime \prime}$ (and sometimes PI from PP), the true RU, NI, and FI. The CA-ME-MI cluster remained highly coherent. None of the last three species were elucidated as a separate identity by genetic fingerprints, as was the case of MI with certain zymograms.

Distinctively polymorphic accessions, which had been previously observed to be enzymatically unique, included 2001, 3001, 6502, and 6503 . New strains to be added to this list included 66 , ENG, 3015, 4030, 4072, 5001, and 7001. Based on preliminary information, there is a high likelihood of additional unique accessions being identified as RFLP research is continued.

MI strains collected in Paraguay over ten years ago were not genetically identical to others collected in 1988 by Drs. Watanabe and Zimmerman. These recent strains were mostly laken from the same semi-arid region (Chaco Province) as the first survey. Certain fingerprints also helped distinguish the cold tolerant RU 6500 series. Further and more extensive trials are necessary in this regard.

The pAm7 MI homologous probe was useful by its discriminatory $(+/-)$ effect with Azolla. The original claim (Plazinski et al., 1989) for MI species specificity was based on dot-blot hybridizations with a few Azolla strains of different species. Our hybridizations with dozens of $\mathrm{Azol}$ la accessions showed that the discrimination was not species-specific (Fig. 2). Successful hybridizations, however, have so far only been achieved with accessions from the CA-ME-MI cluster. On the other hand, accessions of this group also

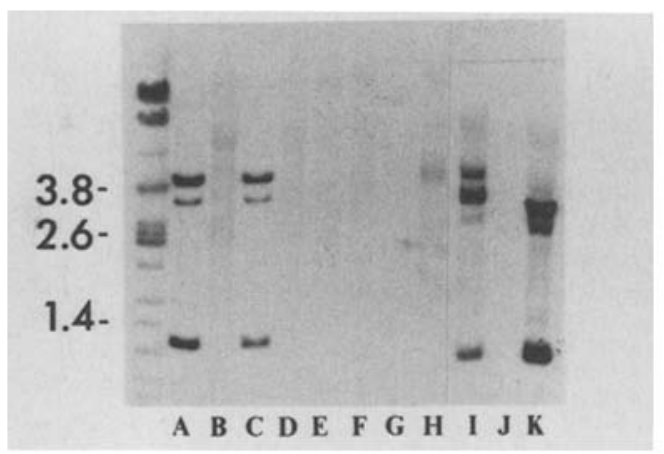

Fig. 2. Southern hybridization with the $\mathrm{pAm} 7$ probe and HindIII-digested DNA of CA and ME accessions.

formed the majority of strains which did not hybridize to pAm7. FI, NI, PI, and PP accessions did not hybridize, but their sample numbers were small. Furthermore, results were unclear for several strains (including RU 6502 and PI 72), so this probe may not be exclusively specific for a select CA-ME-MI subgroup.

\section{Breeding}

Sexual hybrids have been realized from techniques developed for cross-breeding Azolla (Van Cat et al., 1989). FI $\times$ MI hybrids, successfully showing positive heterosis in field biofertilizer studies, are sterile. More recently, fertile hybrids bearing both mega- and microsporangia have been attained by ME $\times$ MI crosses (Watanabe, unpubl.) This achievement provided further evidence of the CA-ME-MI taxonomic relatedness.

Any taxonomic modification of New World species requires more empirical evidence. The answers found in our enzyme and RFLP results are exceeded by the number of new questions derived from our data. Major prerequisites to advancing Azolla genetics are successful utilization of molecular markers, the sporulation trigger, and sexual hybridizations.

\section{Acknowledgements}

The work presented is condensed from a final report of research supported by the U.S. Agency for International Development, Grant no. DPE5542-G-SS-6041-00. 


\section{Azolla diversity}

\section{References}

Maniatis T, Fritsch E F and Sambrook J 1982 Molecular Cloning: A Laboratory Manual. Cold Spring Harbor Press, Cold Spring Harbor, NY.

Plazinski J, Zheng Q, Taylor R, Rolfe B G and Gunning B E S 1989 Use of DNA/DNA hybridization techniques to authenticate the production of new Azolla-Anabaena symbiotic associations. FEMS Microbiol. Lett. 65, 199-204.
Van Cat D, Watanabe I, Zimmerman W J, Lumpkin T A and de Waha Baillonville T 1989 Sexual hybridization among Azolla species. Can. J. Bot. 67, 3482-3485.

Zimmerman W J, Lumpkin T A and Watanabe I 1989a Isozyme differentiation of Azolla Lam. Euphytica 42, 163170.

Zimmerman W J, Lumpkin T A and Watanabe I 1989b. Classification of Azolla spp., section Azolla. Euphytica 43, 223-232. 